\title{
Simple Fabrication Process for Self-Aligned, High-Performance Microscanners- Demonstrated Use to Generate a 2-D Ablation Pattern
}

\author{
Hyuck Choo, David Garmire, James Demmel, Fellow, IEEE, and \\ Richard S. Muller, Life Fellow, IEEE, Member, ASME
}

\begin{abstract}
A new, straightforward, complementary metaloxide-semiconductor (CMOS)-compatible, three-mask process is used to fabricate high-performance torsional microscanners driven by self-aligned, vertically offset comb drives. Both the moving and fixed combs are defined using the same photolithography mask and fabricated in the same device layer, a process allowing the minimum gap between comb fingers to be as small as twice the alignment accuracy of the photolithography process. Our fabricated microscanners have torsional resonant frequencies between $58 \mathrm{~Hz}$ and $24 \mathrm{kHz}$ and maximum optical-scanning angles between $8^{\circ}$ and $48^{\circ}$ with actuation voltages ranging from 14.1 to $67.2 V_{\text {ac-rms }}$. The yields on two separate fabrication runs have been better than $\mathbf{7 0 \%}$. To demonstrate an application for these scanners, we used them to generate laser-ablation patterns suitable for ocular cornea surgery. We assembled a 2-D scanning system by orienting two identical microscanners at right angles to one another. When driven by two $90^{\circ}$ out-of-phase $6.01-\mathrm{kHz}$ sine waves, the cross-coupled scanners produce circular patterns having radii fixed by the amplitude of the driving voltage. Then, we emulated a small pattern from the surface topography found on a U.S. Roosevelt dime and built up an ablation pattern that compares favorably with similar emulations reported by earlier researchers who used larger, more complicated ablation systems. [2006-0185]
\end{abstract}

Index Terms-Complementary metal-oxide-semiconductor (CMOS)-compatible, ocular refractive surgery, self-aligned vertical offset combs, torsional microscanners, 2-D scanning system.

\section{INTRODUCTION}

$\mathbf{M}$ ANY researchers have been motivated to develop simpler, more cost-effective scanner-fabrication technologies because the commercial markets that have resulted from the macroscale torsional scanner (galvano-scanner) technology are mature and lucrative. The scanners are labeled "torsional" because they consist of mirrors supported by beams that are torsionally flexed in order to direct a light beam through an arc. Scanners are essential components for applications such as optical switches in telecommunication

Manuscript received August 31, 2006; revised November 22, 2006. Parts of this paper were presented orally at the 2005 IEEE/LEOS Optical MEMS Conference, Technical Digest 21-22, Oulu, Finland, August 1-4, 2005. This work was supported by the National Science Foundation under the Grants NSFEEC0318642 and CITRIS-NSF-TR22325. Subject Editor H. Fujita.

The authors are with the Electrical Engineering and Computer Sciences Department, University of California at Berkeley, Berkeley, CA 94720-1774 USA (e-mail: hchoo@eecs.berkeley.edu).

Digital Object Identifier 10.1109/JMEMS.2007.895048 networks [1], high-definition and retinal displays for entertainment, engineering, and educational markets [2]-[5], barcode scanning for inventory monitoring, endoscopic, confocal, and coherent tomography imaging in biomedical fields [6]-[8], range-finding systems for safe vehicular navigation, and free space laser communications [9]

A number of previous researchers have used the methods that are generally identified as "microelectromechanical system (MEMS) technologies" to fabricate microscanners in order to exploit the precise design and mass production advantages afforded by this choice. Specific results as reported for earlier research demonstrate, however, that there are pitfalls that lead to undesirable complexity, lowered processing yields, and other difficulties. A major problem area is centered on actuation of the microscanners.

Torsional microscanners typically require substantial torque and to produce this torque by electrostatic drivers, previous research [10] has shown the effectiveness of using vertically offset comb pairs for the drivers. Fabrication of these vertically offset combs has been a considerable challenge to earlier MEMS technologists, who have found the solutions using the following demanding procedures: 1) carrying through critical alignment steps in a two-wafer process [10], 2) controlling and replicating the properties of materials such as photoresist or bimorph layers so that these materials can function as hinges [11], 3) postprocess annealing in a high-temperature furnace following the hand assembly of lids on device chips [12], and 4) depositing multiple-masking layers (composed of silicon dioxide and silicon nitride) [13] to create the offset combs. We decided to focus our research on the development of fabrication methods that would produce vertically offset comb pairs using more conventional integrated-circuit (IC) processing tools.

This paper reports a complementary metal-oxide-semiconductor (CMOS)-compatible MEMS technology that we have developed and demonstrated for batch-fabricating high-performance torsional microscanners. Our microscanner-fabrication technology employs only conventional silicon-processing tools that have proven their effectiveness and user-friendliness through large scale use in the integrated circuits industry. The required temperatures for all of our processing steps are low enough to allow prefabrication of CMOS electronics directly on the same wafer as the microscanner devices. The yield for this new scanner process has exceeded $70 \%$ on two fabrication 


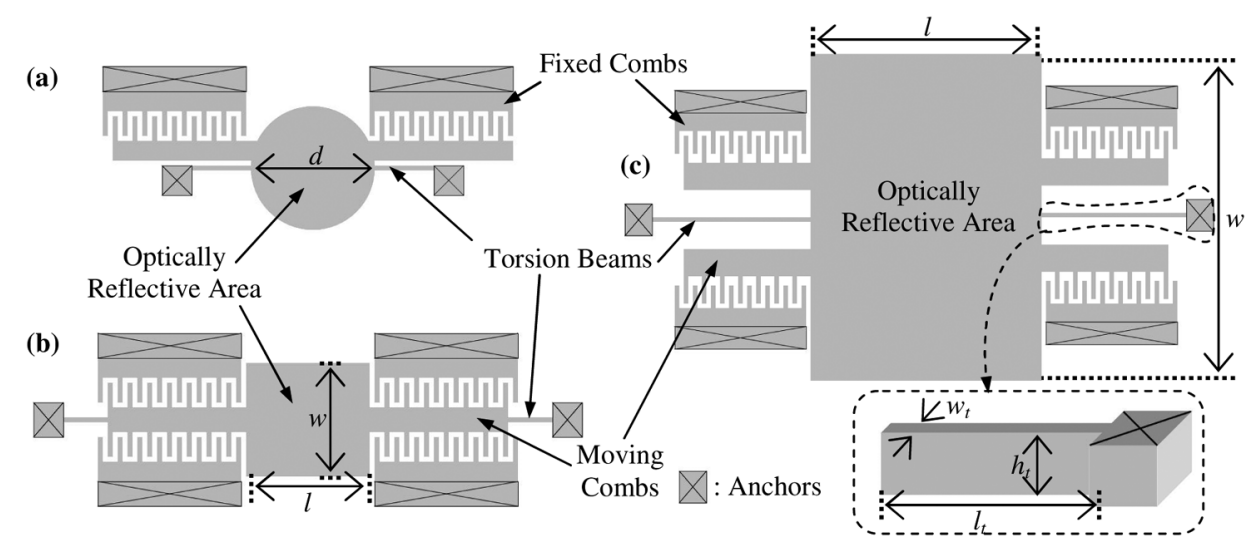

Fig. 1. Three different microscanner designs: (a) fast microscanner with a circular scanning area (or optically reflective area); (b) very fast microscanner with a rectangular scanning area; and (c) slow microscanner with an extra large scanning area; inset at lower right is a plan view showing the dimensions of the microscanner support beams.

runs (116 devices per wafer, 2 wafers per run) made in the University of California at Berkeley Microlab.

Operational tests on the fabricated microscanners show that they easily meet the performance requirements for many applications in the biomedical, telecommunication, and imaging systems areas. To demonstrate the microscanner performance, we have investigated a particular application for which we find our design to be well suited: refractive laser surgery of ocular corneas where small spot size and high scan speeds are important assets [14]. In ocular refractive surgery, surgeons need to steer and control laser pulses to reshape a patient's corneas in order to improve his vision. To demonstrate this use with our microscanners, we have employed them to generate ablation-emulation results that are superior to published emulation patterns that had been produced by commercial, state-of-the-art eye-surgery microscanners [14].

There are potentially many additional MEMS applications for the robust, high-performance comb drivers introduced in this paper. As a result of their fabrication using only conventional IC processing tools, there is excellent control of critical dimensions such as comb-finger spacings. These spacings are determined by a single photomasking step which allows them to be as small as two times the alignment accuracy of the photolithography process (which is $2 \times 7 \mathrm{~nm}=14 \mathrm{~nm}$ for Nikon NSR-S609B). However, the practical minimum gap sizes are typically $1 \mu \mathrm{m}$ wide or larger because they are determined by the fabrication process limitations and variations such as an achievable aspect ratio of a deep reactive ion etching (DRIE) process as well as the sidewall erosions commonly observed in plasma-etch processes. Minimizing gap spacing reduces the driving voltage needed to provide a given force. As an example, we consider a typical design in which vertical combs having gaps of $3 \mu \mathrm{m}$, widths of $5 \mu \mathrm{m}$, and lengths of $100 \mu \mathrm{m}$, are laid out using $25 \%$ of the comb-drive area for supporting structures. With this design, finished combs will exert an out-of-plane force density of $13.8 \mu \mathrm{N} / \mathrm{cm}^{2} / \mathrm{V}^{2}$. Comb drives having this force density can be used advantageously in many ways; for example, in adaptive optics for mirror-curvature adjustments [15], in vertical inch-worm motors [16], in phase-shifting interferometers [17], and also, in acoustic speakers. In yet another application, by producing a capacitance change of $27.6 \mathrm{pF} / \mathrm{cm}^{2}$ per micrometer of out-of-plane motion, the combs can gainfully be applied to the design of $z$-axis accelerometers, to innovative microphone technologies and to microstage positioning systems.

\section{DESIGN, FABRICATION, AND CHARACTERIZATION}

\section{A. Microscanner Design}

We have investigated three different designs of microscanners as pictured in Fig. 1 .

For each of these three designs, we have varied the dimensions (diameters of circular reflective areas or lengths and widths of rectangular reflective areas) of the optically reflective areas as along with the lengths and the widths of the torsion beams. To predict the resonant frequencies of these designs, we calculate the torsional stiffness of the beams using Timoshenko's equation [18]

$$
\begin{aligned}
& k_{t}= \frac{1}{3} G\left(w_{t}\right)^{3}\left(\frac{h_{t}}{l_{t}}\right) \\
& \times\left[1-\frac{199}{\pi^{5}} \frac{w_{t}}{h_{t}} \sum_{n=1,3,5 \ldots}^{\infty} \frac{1}{n^{5}} \tanh \left(\frac{n \cdot \pi \cdot h_{t}}{2 w_{t}}\right)\right], \\
& h_{t}>w_{t} .
\end{aligned}
$$

In (1), $G$ is the torsional modulus for silicon, and $w_{t}$ and $h_{t}$ are the width and height of the beam, as indicated in the bottomright inset of Fig. 1. The microscanner resonant frequency is given by

$$
f_{r}=\frac{1}{2 \pi} \sqrt{\frac{k_{t}}{u_{m}}}
$$

where $I_{m}$ is the mass-moment of inertia of the microscanner (given in Table II), the value of which depends on the microscanner geometry and is readily calculated [19].

Table I lists the dimensions of the microscanner embodiments investigated and their predicted resonant frequencies [using (2)]. By making various combinations of reflective areas of different sizes and torsion beams of differing dimensions (resulting in different torsional stiffnesses) as listed in Table I, we designed microscanners having predicted resonant frequencies ranging 
TABLE I

PARAMETER VARIATIONS FOR MICROSCANNER EMBODIMENTS

\begin{tabular}{|c|c|c|c|}
\hline & $\begin{array}{l}\text { Large Rectangular } \\
\text { Microscanners }\end{array}$ & $\begin{array}{c}\text { Circular } \\
\text { Microscanners }\end{array}$ & $\begin{array}{c}\text { Rectangular } \\
\text { Microscanners }\end{array}$ \\
\hline $\begin{array}{l}\text { Dimensions: } \\
\text { Reflective } \\
\text { Area* }^{*}\end{array}$ & $\begin{array}{c}l: 3 \mathrm{~mm} \\
w: 6,7, \text { or } 8 \mathrm{~mm} \\
t_{\text {sor }}: 50 \mu \mathrm{m}\end{array}$ & $\begin{array}{c}d: 0.5 \text { or } 1 \mathrm{~mm} \\
t_{\text {Sor }}: 30 \mu \mathrm{m}\end{array}$ & $\begin{array}{c}l: 1,1.5, \text { or } 3 \mathrm{~mm} \\
w: 0.5,1, \text { or } 1.5 \mathrm{~mm} \\
t_{\text {soI }}: 50 \mu \mathrm{m}\end{array}$ \\
\hline $\begin{array}{l}\text { Dimensions: } \\
\text { Torsion } \\
\text { Beams }\end{array}$ & $\begin{array}{c}\boldsymbol{l}_{\boldsymbol{i}}: 1 \mathrm{~mm} \\
\boldsymbol{w}_{\boldsymbol{t}}: 10,15,20, \text { or } 30 \mu \mathrm{m} \\
\boldsymbol{h}_{\boldsymbol{t}}: 25 \text { or } 50 \mu \mathrm{m}\end{array}$ & $\begin{array}{c}\boldsymbol{l}_{\boldsymbol{t}}: 0.2,0.3,0.4, \text { or } 0.5 \mathrm{~mm} \\
\boldsymbol{w}_{\boldsymbol{t}}: 10 \text { or } 20 \mu \mathrm{m} \\
\boldsymbol{h}_{\boldsymbol{t}}: 30 \mu \mathrm{m}\end{array}$ & $\begin{array}{c}\boldsymbol{l}_{i}: 0.4,0.6,0.8, \text { or } 1 \mathrm{~mm} \\
\boldsymbol{w}_{\boldsymbol{t}}: 33.3,40, \text { or } 50 \mu \mathrm{m} \\
\boldsymbol{h}_{\boldsymbol{t}}: 50 \mu \mathrm{m}\end{array}$ \\
\hline $\begin{array}{c}\text { Moment } \\
\text { of } \\
\text { Inertia* }\end{array}$ & $\frac{\rho_{S i} \cdot t_{S O I} \cdot l \cdot w^{3}}{12}$ & $\frac{\pi \cdot \rho_{S i} \cdot t_{S O I} \cdot d^{3}}{64}$ & $\frac{\rho_{S i} \cdot t_{S O I} \cdot l \cdot w^{3}}{12}$ \\
\hline Predicted $\boldsymbol{f}_{r}$ & $50-230 \mathrm{~Hz}$ & $1-10 \mathrm{kHz}$ & $4-26 \mathrm{kHz}$ \\
\hline
\end{tabular}

${ }^{*} t_{S O I}$ : Thickness of the device layer of SOI wafer; and $\rho_{S i}$ Density of silicon $\left(2330 \mathrm{~kg} / \mathrm{m}^{3}\right)$

Handling Layer of sol Wafer

Device Layer of SOI Wafer

Photoresist

Buried Oxide

Thermal Oxide

(Or LTO)

LTO
Top View
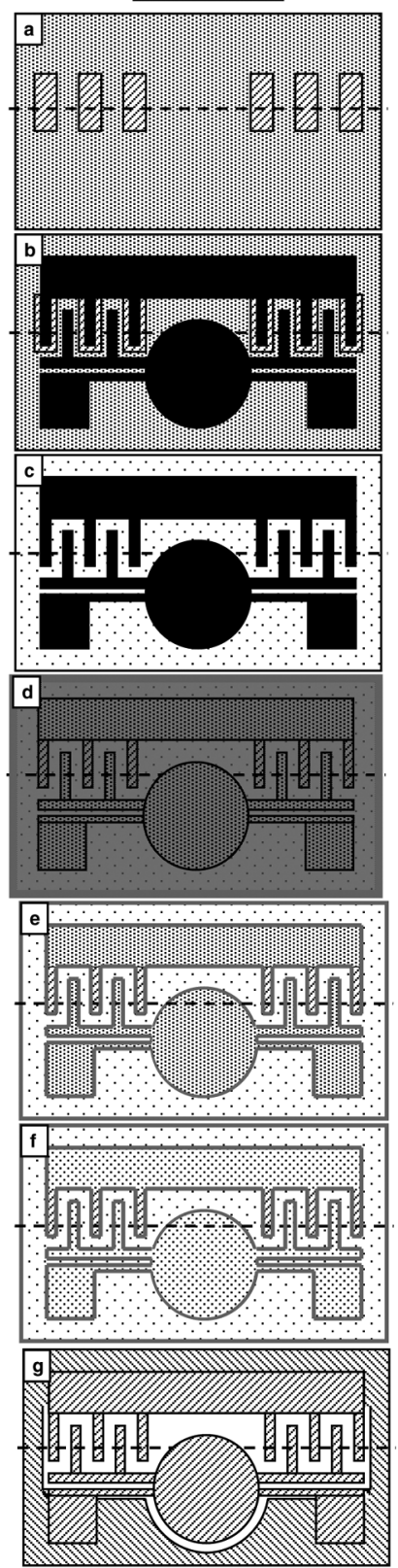

Cross-section View
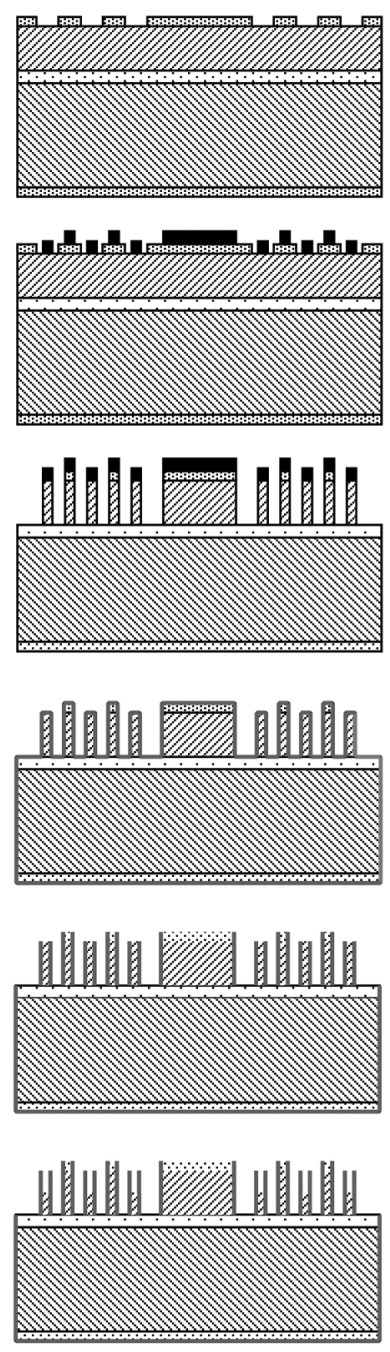

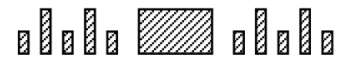

Fig. 2. Fabrication process for torsional microscanners with self-aligned, vertically offset combs. The left column shows top views while the right column shows cross-sectional views along the dotted lines following completion of the processing steps described in Section II-B. 


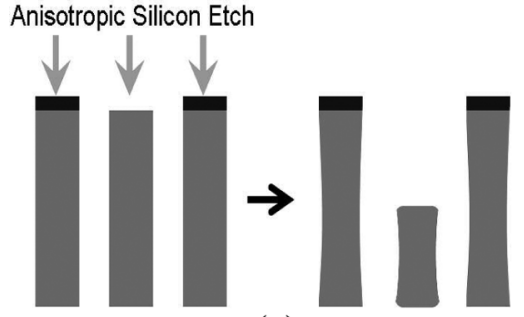

(a)

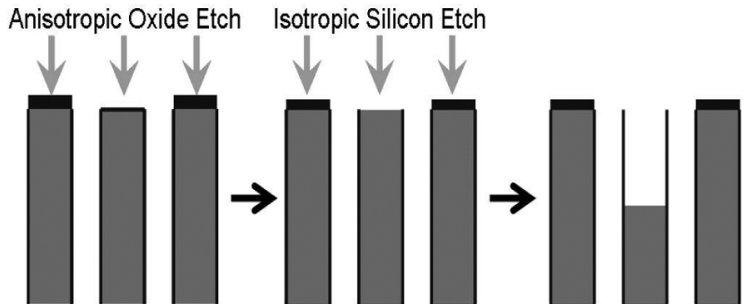

(b)

Fig. 3. Etch methods to produce offsets to the comb fingers using (a) anisotropic silicon etch and (b) oxide layer and isotropic silicon etch.

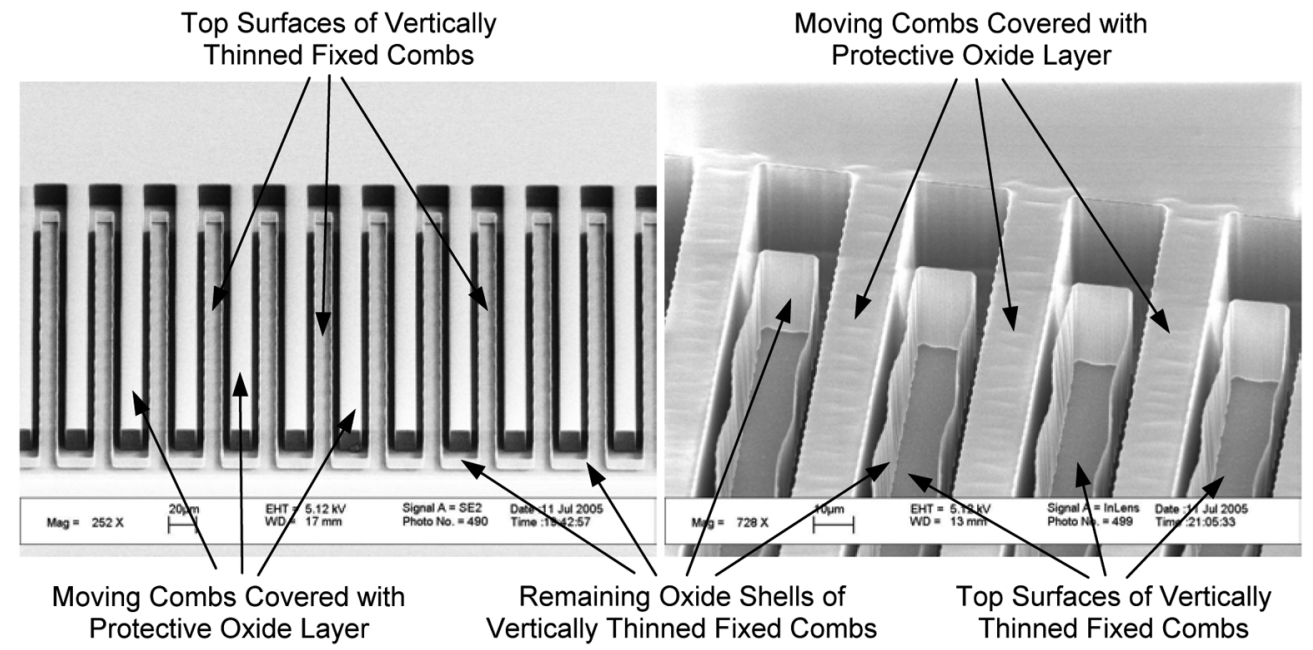

Fig. 4. SEM images of moving- and fixed-comb fingers after completing fabrication step f) [Fig. 2(f)]. The upper two images show offset combs being processed on SOI wafers with a 30- $\mu \mathrm{m}$ device layer (offset height: $15 \mu \mathrm{m}$ ) while the lower two images show offset combs being processed on SOI wafers with a 50- $\mu \mathrm{m}$ device layer (offset height: $25 \mu \mathrm{m}$ ).
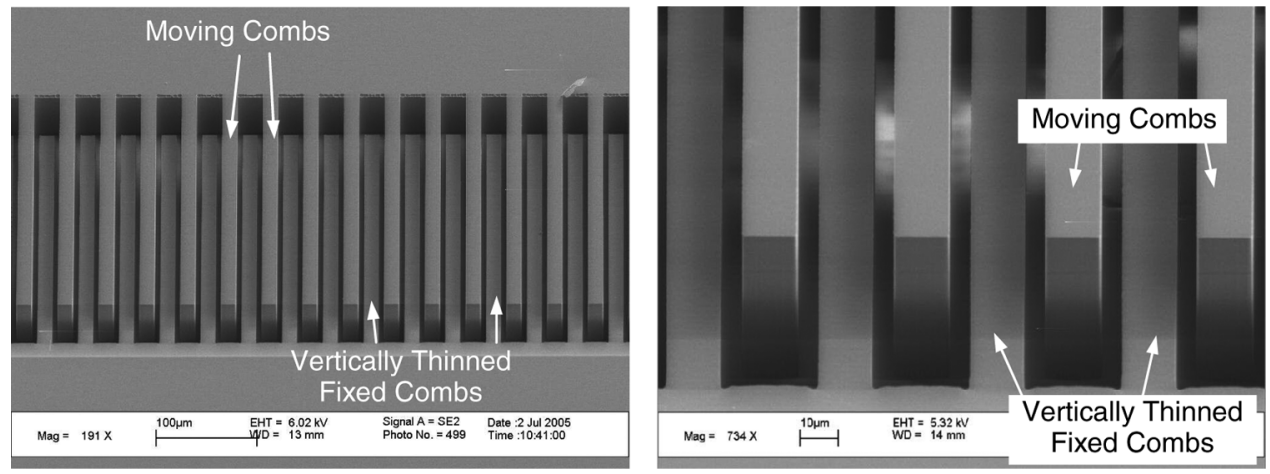

Fig. 5. SEM images of moving- and fixed-comb fingers after HF release [process step g)]. The completely processed comb fingers have sharp, well-defined rectangular shapes with very smooth surfaces, regardless of having undergone the silicon isotropic etch step or not.

\section{3-D Profile (WYKO Measurement)}

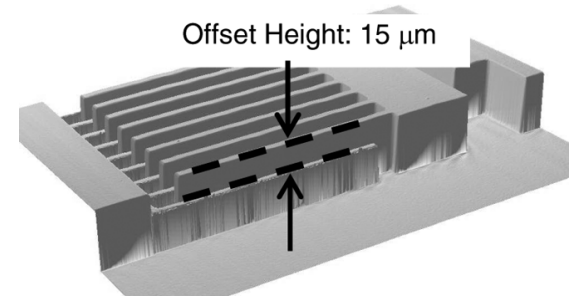

Fig. 6. The 3-D profile measurements of vertically offset combs and the uniformity of offset heights at five locations on a $10-\mathrm{cm}$ processed wafer: The measurements were made at its center and at locations, $2 \mathrm{~cm}$ from the outer edge of the wafer. The values are peak-to-peak deviations from the average value.
TABLE II

DeViation From the Average Value $(15 \mu \mathrm{m})$

\begin{tabular}{|c|c|c|c|c|}
\hline Center & Left & Right & Top & Flat \\
\hline$+1.53 \%$ & $-1.38 \%$ & $+0.13 \%$ & $+0.90 \%$ & $-0.28 \%$ \\
\hline$+0.23 \mu \mathrm{m}$ & $-0.21 \mu \mathrm{m}$ & $+0.02 \mu \mathrm{m}$ & $+0.14 \mu \mathrm{m}$ & $-0.04 \mu \mathrm{m}$ \\
\hline
\end{tabular}

from $50 \mathrm{~Hz}$ to $26 \mathrm{kHz}$. This wide range of resonant frequencies can address the requirements for microscanners having many different applications. 
(a)

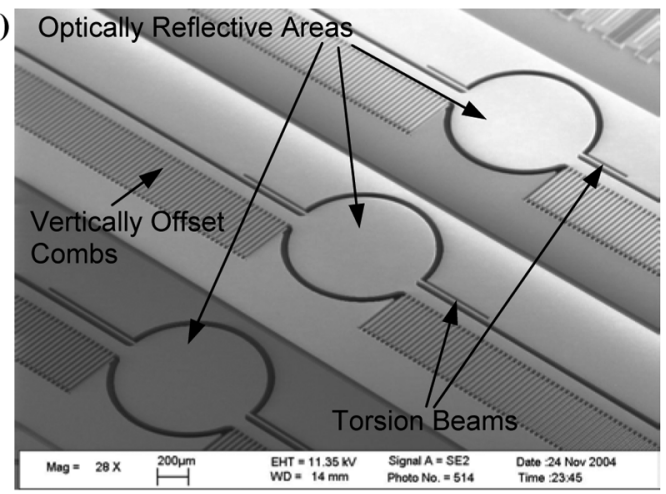

(b)

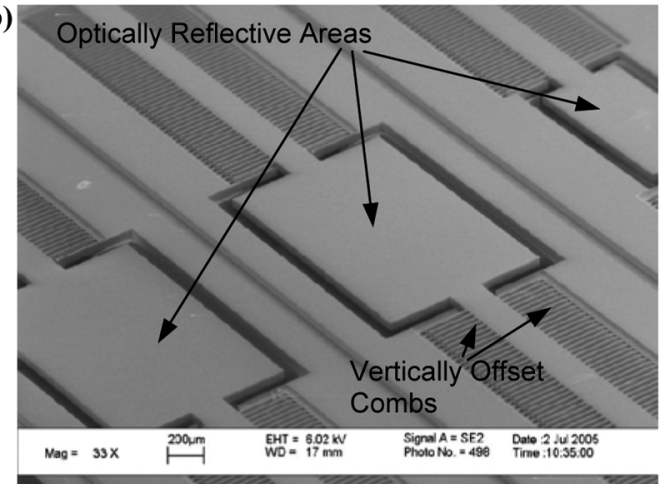

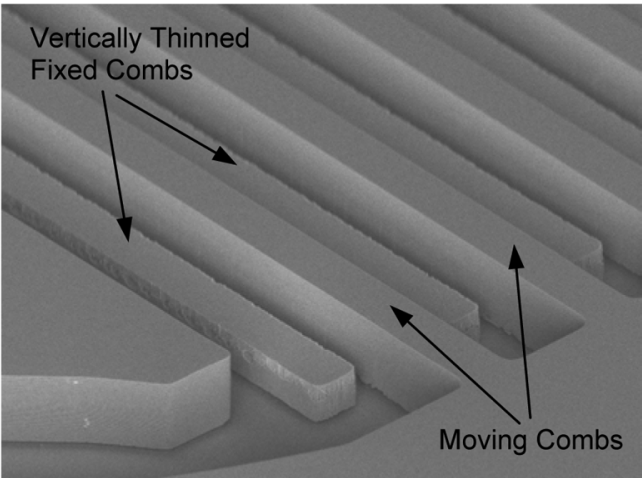

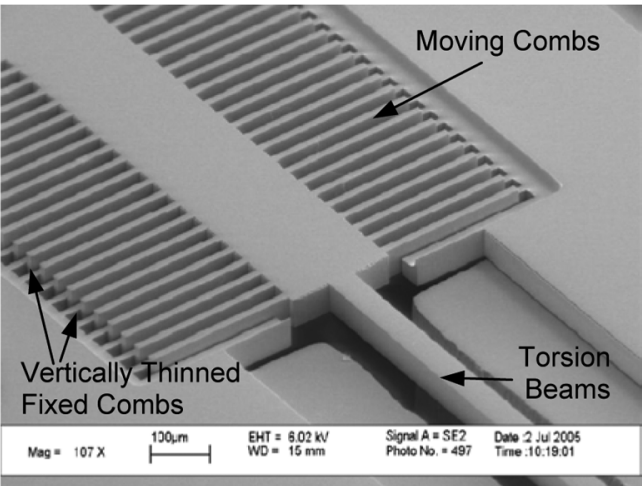

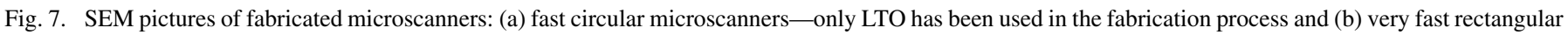
microscanners-only thermal oxide has been used in the fabrication.

TABLE III

RESONANT-MOTION PROPERTIES OF REPRESENTATIVE MicRosCANNERS

\begin{tabular}{|c|c|c|c|c|}
\hline $\boldsymbol{f}_{\boldsymbol{r}}$ & $\boldsymbol{Q}$ & OSA & $\mathbf{V}_{\mathrm{ac}}(\mathbf{r m s})$ & Dimensions \\
\hline $58 \mathrm{~Hz}^{*}$ & 40 & $20.8^{\circ}$ & 14.1 & $\begin{array}{c}\boldsymbol{l}=3 \mathrm{~mm} \\
\boldsymbol{w}=8 \mathrm{~mm}\end{array}$ \\
\hline $6.01 \mathrm{kHz}^{* *}$ & 67 & $24.2^{\circ}$ & 26.4 & $\begin{array}{c}\boldsymbol{d}=1 \mathrm{~mm} \\
\text { (Circular Microscanner) }\end{array}$ \\
\hline $8.89 \mathrm{kHz}^{* *}$ & 70 & $22^{\circ}$ & 34.9 & $\begin{array}{c}\boldsymbol{d}=1 \mathrm{~mm} \\
\text { (Circular Microscanner) }\end{array}$ \\
\hline $12.5 \mathrm{kHz}^{* * *}$ & 180 & $48^{\circ}$ & 67.2 & $\begin{array}{c}\boldsymbol{l}=1.5 \mathrm{~mm} \\
\boldsymbol{w}=1 \mathrm{~mm}\end{array}$ \\
\hline $24 \mathrm{kHz}^{* * *}$ & 300 & $17^{\circ}$ & 35.2 & $\begin{array}{c}\boldsymbol{l}=1 \mathrm{~mm} \\
\boldsymbol{w}=0.5 \mathrm{~mm}\end{array}$ \\
\hline
\end{tabular}

*: Slow large rectangular microscanner; **: Fast circular microscanners; ***: Very fast rectangular microscanners

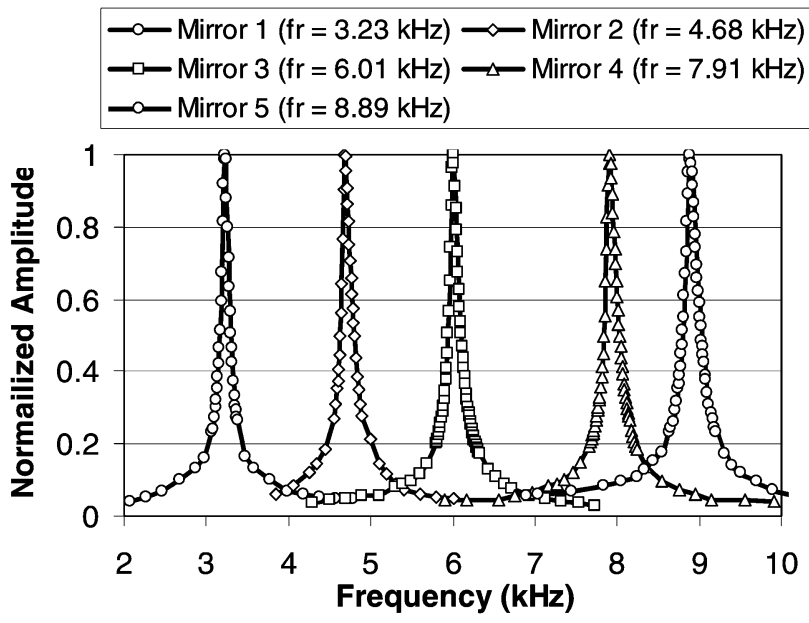

Fig. 8. Frequency response of selected microscanners with circular reflective areas.

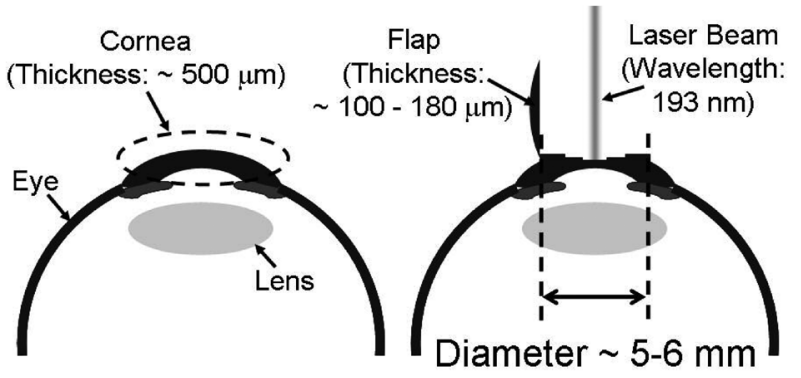

Fig. 9. Ocular refractive surgery: Using a microkeratome (a very fine blade), a corneal flap is cut open. Then, using a laser (wavelength: 193-208 nm), the exposed inner tissue of the cornea is selectively ablated to correct optical aberrations and thereby improves the patient's vision.

\section{B. Microscanner Fabrication}

The microscanner fabrication process, which involves the use of three photolithography masks [two for defining features in the device layer of a silicon-on-insulator (SOI) wafer and one for opening the backside of the microscanners], is illustrated in Fig. 2. The following is true.

a) We start with a $\langle 100\rangle n$-type SOI wafer (device-layer thickness: 30 or $50 \mu \mathrm{m}$, resistivity: $0.005-0.01 \Omega \mathrm{cm}$ ) and grow $0.5-\mu \mathrm{m}$ thermal oxide at $900{ }^{\circ} \mathrm{C}$ or deposit lowtemperature oxide (LTO) at $400^{\circ} \mathrm{C}$. LTO must be chosen if it is necessary to ensure that the fabrication process remains completely CMOS-compatible. Because the oxide layers serve only as protective layers, the LTO layer does not need to go through a densification process at high temperatures (which would typically exceed $950^{\circ} \mathrm{C}$ ). Next, using the photolithography mask $\# 1$, we pattern and remove the 
(a)

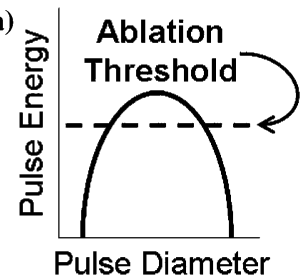

(b)

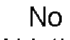

Ablation

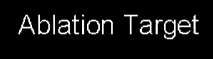

(c)

Single

Ablation
Ablation Target

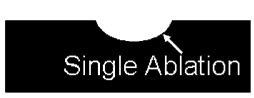

(d)

Two

Ablations

(e)

Multiple

Ablations

Fig. 10. Summary of ablation process for refractive surgery: (a) only that part of the laser pulse having energy density above an ablation-threshold value will ablate the target; (b) no ablation performed; (c) ablation pattern generated by applying a single laser pulse having the pulse-energy distribution shown in (a); (d) ablation pattern generated by applying two subsequent laser pulses; and (e) spherical profile generated by applying multiple, coordinated laser pulses.

oxide (thermal or LTO) selectively where fixed combs will be later fabricated and vertically thinned [Fig. 2(a)].

b) Using mask \#2, we define patterns for the microscanners, including moving/fixed combs, flexures, and the geometries of the reflective area, on the top surface of the device layer [Fig. 2(b)]. The fixed combs must be defined within the windows from which the oxide has been removed to expose the silicon surface in the previous step, and the minimum gap between the moving and fixed-comb fingers can be as small as twice the alignment accuracy of the photolithography system.

c) After hard-baking the patterned photoresist at $120^{\circ} \mathrm{C}$ for one hour, we first perform anisotropic oxide etch, and then, use DRIE to pattern the microscanner structures (including the optically reflective area, comb fingers, and flexures) in the device layer of the SOI wafer [Fig. 2(c)].

d) Once the DRIE etching is complete, we remove the photoresist layer and deposit a very thin layer $(\sim 0.2 \mu \mathrm{m})$ of thermal oxide or LTO, in order to stop erosion of the sidewalls of the structures that were created in the previous step [Fig. 2(d)]. After this thin oxide has been deposited, there are $0.2-\mu \mathrm{m}$-thick oxide layers on top of the fixed-comb fingers and approximately $0.7-\mu \mathrm{m}$-thick oxide layers on all other surfaces including, especially, the top surfaces of the movable comb fingers and of optically reflective surfaces.

e) Following the oxide growth (or deposition), we perform a timed anisotropic-plasma-oxide etch to remove the $0.2-\mu \mathrm{m}$-thick oxide from the top-facing surfaces. This step exposes the silicon surface on top of the fixed combs, but leaves all other surfaces covered by an approximately 0.5 - $\mu$ m-thick oxide layer [Fig. 2(e)].

f) In a next step, we use a timed plasma etch that erodes silicon isotropically to etch the exposed top surfaces of the fixed combs, thinning only these fingers because all other surfaces of the structures are still protected by an oxide layer [see Figs. 2(f), 3(b), and 4].

g) The, using mask \#3, we pattern and open the backside of the microscanners, and release the devices in concentrated high-frequency (HF) followed by critical point drying (CPD) [Fig. 2(g)].

Comment on the need for steps $d$ ) and f), thin-oxide deposition and selective removal: In theory, the previously described process might be simplified through omission of steps $d$ )-f), replacing them by an anisotropic etch which should erode only the exposed fixed-comb tops. In practice, we have found, however,

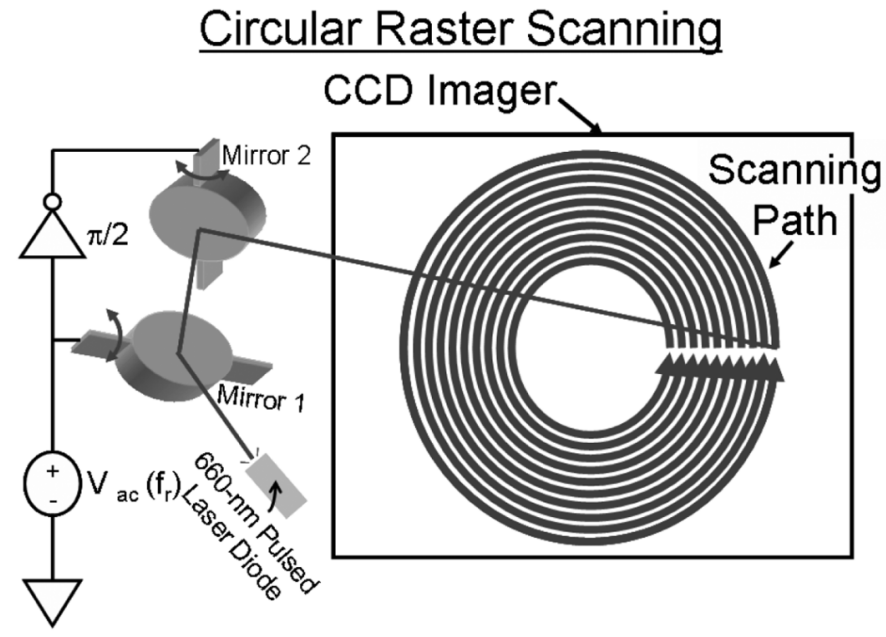

Fig. 11. The 2-D scanning system realized using a pair of identical microscanners.

that anisotropic silicon etch does, in fact, erode surfaces that are not perpendicular to the beam causing considerable silicon removal from the comb sidewalls.

This erosion leads to unacceptable control of comb-finger widths and of the gaps separating the fingers. Accordingly, we have added the thin-oxide deposition and selective removal steps to produce our microscanners.

Fig. 4 shows scanning electron microscope (SEM) images of moving-and fixed-combs taken immediately following completion of the timed-isotropic silicon etch described in process step f) and shown in Fig. 2(f). The vertically etched top surfaces as well and the remaining oxide shells are clearly visible. Compressive stress in the silicon dioxide layer is the source for the waviness of the vertical oxide shell. The oxide waviness does not have any effect on the final shape of the silicon comb fingers, as shown in Fig. 5.

Fig. 5 shows SEM images of released combs [following step g)]; the comb fingers are clearly vertically offset, sharply defined, and precisely aligned. The top surfaces of the isotropically etched fixed-comb fingers are visually as smooth as the surfaces of the adjacent unprocessed comb fingers that form the moving-comb pair.

Using a WYKO NT3300 optical surface profiler, we measured the offset heights of vertically offset comb fingers around the wafers. The measurements were made at five locations, $2 \mathrm{~cm}$ from the outer perimeter of $10-\mathrm{cm}$ processed wafers (Fig. 6). The peak-to-peak deviations at five locations shown 


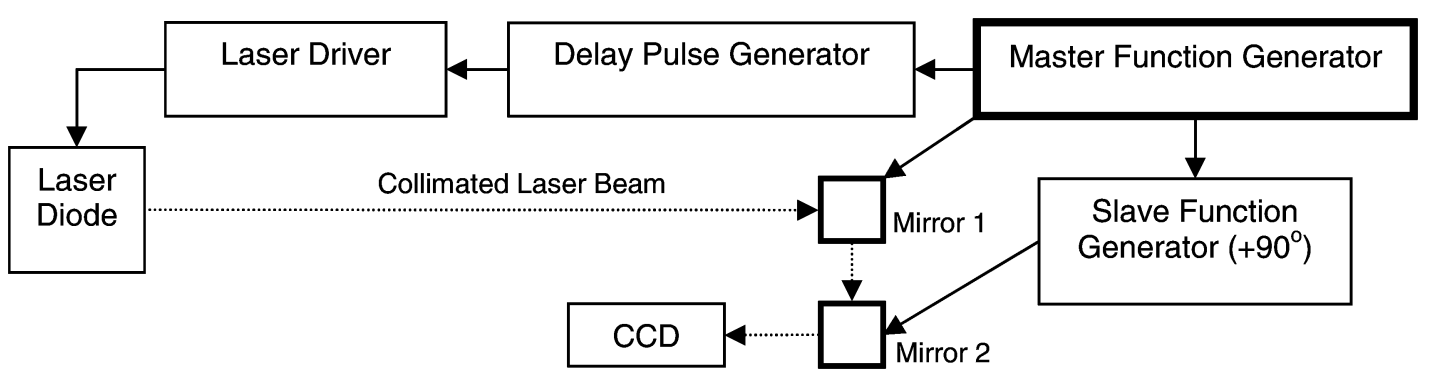

Fig. 12. Schematic diagram of experimental setup using scanners to generate cornea ablation patterns.

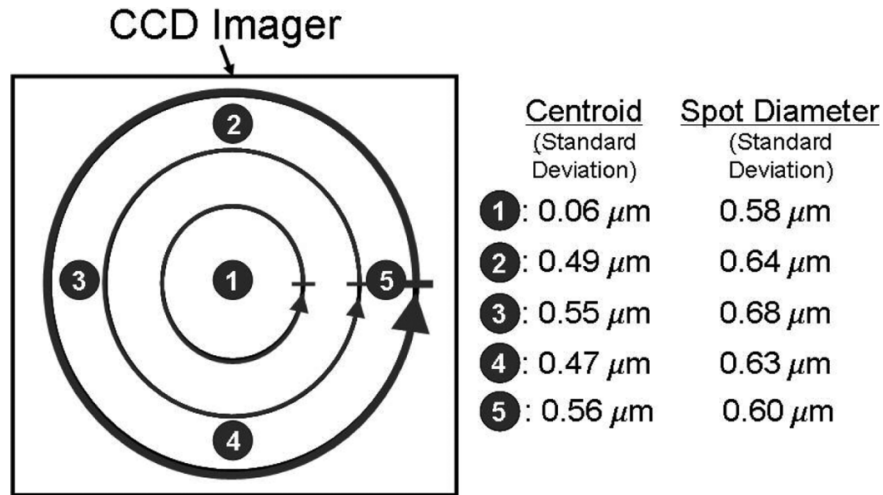

Fig. 13. Stability and repeatability of our 2-D scanning system within $30 \mathrm{~min}$.

in Table II are smaller than the thickness fluctuations found stated in the specifications for the device layers of SOI wafers (typically $0.5-2 \mu \mathrm{m}$ ), and they show excellent uniformity for the new process.

\section{Fabricated Microscanners}

SEM pictures of fabricated microscanners are shown in Fig. 7.

Our fabrication process has produced high yields on two separate fabrication runs (116 micromirrors per wafer, two wafers per run). Between $70 \%$ and $85 \%$ of tested microscanners perform properly on all of the wafers. Damages to microscanners mostly occurred during step g), the final HF-release/rinsing/critical-point-drying step as a consequence of rough handling.

Table III shows the resonant frequencies $\left(f_{r}\right)$, quality factors $(Q)$, and maximum optical scan angles (OSA) at resonance measured for selected microscanners driven with sine waves having the tabulated root-mean-square (rms) amplitudes. Here, OSA is defined as an angle that is twice the mechanical scan angle that a microscanner physically rotates. Resonant frequencies of the fabricated microscanners ranged from $58 \mathrm{~Hz}$ to $24 \mathrm{kHz}$. The maximum resonant amplitudes achieved by the microscanners ranged from $8^{\circ}$ to $48^{\circ}$, with most microscanners exhibiting OSA of $20^{\circ} \pm 5^{\circ}$. The actuation voltages ranged from 14.1 to $67.2 V_{\text {ac_rms. }}$. Fig. 8 shows the frequency response measured for five different microscanners.

\section{Microscanner Application Example: Emulating A Cornea Ablation Process}

In order to evaluate our microscanners in a "real-world" application, we investigated their possible application to the task of refractive laser surgery of ocular corneas. Scanners used for this purpose should be effective in steering very small laser beams at high scan speeds [14]. The purpose of the surgery is to reshape optical corneas by ablating tissue in order to correct optical aberrations as shown in Fig. 9.

Ablation of human corneas is a cumulative process, as shown in Fig. 10. Only the section of the laser pulse having an energy level higher than the threshold value $\left(50-60 \mathrm{~mJ} / \mathrm{cm}^{2}\right)$ causes ablation [14], [20]. The depth of the ablated tissue is proportional to the logarithm of the laser-pulse energy [20].

To demonstrate this application, we assembled a 2-D scanning system by orienting two identical microscanners at right angles to one another as shown in Fig. 11 (mirror \#3 in Fig. 8, mirror diameter $=1 \mathrm{~mm}$, resonant frequency $=6.01 \mathrm{kHz}$ ) and scanned a pulsed laser beam (670-nm wavelength). The crosscoupled scanners were driven by two $6.01-\mathrm{kHz}$ sine waves that were $90^{\circ}$ out of phase, producing circular patterns having radii fixed by the amplitude of the driving voltage with an intensity governed by the modulated laser (Fig. 12). For cornea ablation, circular scanning provides for an excellent match to the cornea's geometry and is, therefore, favorable over the more typical raster scanning which uses linear sweeps by horizontaland vertical-scanning mirrors to trace out a pattern.

In the ablation system, laser spots forming the pattern persist for $0.4 \mu \mathrm{s}$ and have a $220-\mu \mathrm{m}$ diameter (full width/half maximum) as measured with a charge coupled devices (CCD) optical sensor. The wavelength of the laser is $660 \mathrm{~nm}$. A CCD sensor, positioned in place of the ocular cornea, allows us to assess performance of the system. As mentioned earlier, refractive laser surgery is a cumulative ablation process [14]. To mimic the real process, we capture the scanning pattern at each CCD frame and then sum the intensity profiles which are proportional to the final ablation pattern. The usual period of time for optical laser surgery is shorter than $20 \mathrm{~min}$ so we measured the repeatability and stability of our system over a period of $30 \mathrm{~min}$ (Fig. 13). Our system demonstrates excellent repeatability in pulse position (standard deviation less than $0.56 \mu \mathrm{m}$ ) as well as in pulse diameter (standard deviation less than $0.68 \mu \mathrm{m}$ ) around the ablation zone.

To demonstrate the versatility of our area scanner, we have emulated a small pattern from the surface topography found on a U.S. Roosevelt dime. The 3-D topology of the region of interest on the United States dime was measured using a WYKO NT3300 and is shown in Fig. 14(b) and (c) as well as in Fig. 15(a). The height information was then converted into a grayscale image (0-255 level), which is easier to utilize for ablation process. 

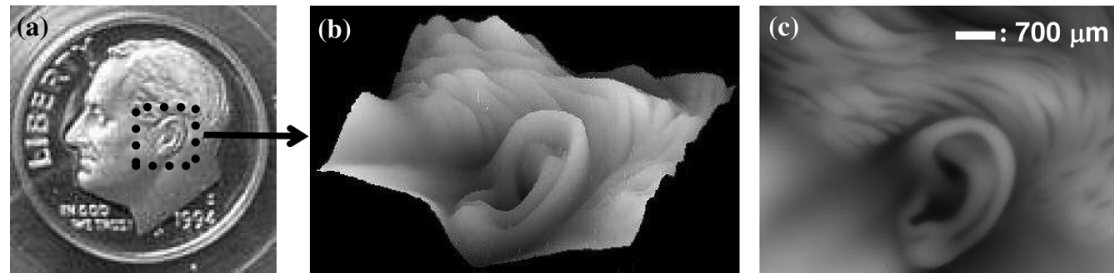

Fig. 14. Selected ablation target pattern: (a) picture of a United States dime; (b) 3-D profile of the region of interest indicated by the dotted circle (measurements by using WYKO NT 3300); and (c) 3-D profile converted to a grayscale image (based on height information), which is more convenient for emulating the ablation process.
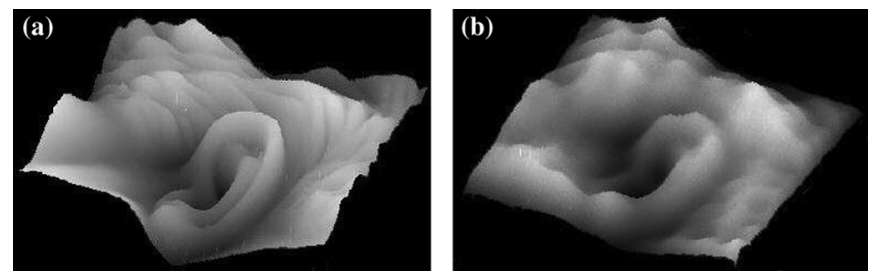

Fig. 15. (a) WYKO 3-D surface profile [Fig. 14(b) repeated]. (b) The 3-D diagram of small-spot ablation replica of the dime surface captured by our microscanner and CCD system (peak-to-valley height difference is approximately $93 \mu \mathrm{m}$ on the original surface).
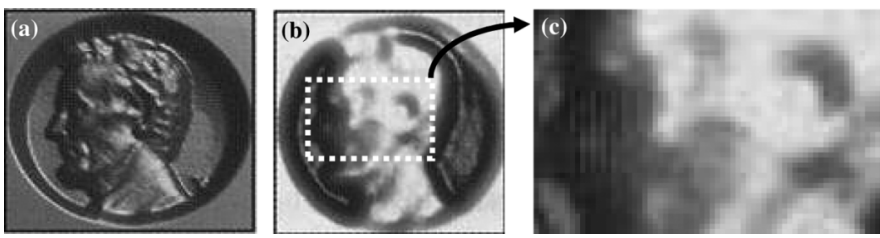

Fig. 16. Laser-ablation pattern produced by a commercially available system: (a) surface topology of a United States penny (target pattern); (b) reproduced pattern using a state-of-the-art refractive surgery system; (c) magnified image zoomed in around the ear of the reproduced pattern (after [14]). Fig. 15(b) can be compared to Fig. 16(c) for an evaluation of performance of our ablation scanning system with the performance of a commercial system (VISX STAR S4).

Next, according to the grayscale image, we have built up an ablation pattern over a 40-min interval. The resultant pattern is shown in Fig. 15(b). Because the scanning spot was $220 \mu \mathrm{m}$ in diameter, some of the very fine details were lost. Yet, overall, the emulated ablation image contains many details and shows good-quality depiction of the original target pattern.

Fig. 16 shows a similar result presented by researchers to demonstrate their ablation capabilities using a state-of-the-art refractive surgery system [14]. By comparing Fig. 15(b) with the images shown in Fig. 16(b) and (c), we can judge at least qualitatively that the microscanner ablation system that we have presented performs very well as compared to presently available macrosized tissue-ablation systems.

\section{CONCLUSION}

We have designed, fabricated, and tested microscanners using our new fabrication techniques. The process uses well-developed integrated-circuit processing tools, and is simple, high yielding, and reliable. The major advance in our fabrication process results from its straightforward method to produce vertically offset comb pairs that provide for robust electrostatic drive of torsion-bar suspensions. In practice, we achieve uniform offset heights for vertical comb fingers processed across the $10-\mathrm{cm}$ wafers. We have produced microscanners having resonant frequencies ranging from $50 \mathrm{~Hz}$ to $24 \mathrm{kHz}$ having OSA values typically approximating $20^{\circ}$ but varying from $8^{\circ}$ to $48^{\circ}$. The actuation voltages required were from 14.1 to $67.2 V_{\text {ac_rms. }}$.

A 2-D scanning system, built using these microscanners, produced emulated ablation patterns that compare favorably to results published by researchers to illustrate the performance of a state-of-the-art macroscale ablative surgery system.

\section{ACKNOWLEDGMENT}

The authors would like to thank M. Wasilik of University of California Berkeley Microlab for developing silicon etch recipes and Dr. M. Helmbrecht of Iris Adaptive Optics (I. AO, Berkeley, CA) for the loan of a high-voltage amplifier.

\section{REFERENCES}

[1] M. Ward and F. Briarmonte, "Lucent's new all-optical router uses bell labs microscopic mirrors," Bell Labs Innovations in the News Nov. 1999 [Online]. Available: http://www.bell-labs.com/news/1999/ november/10/1.html

[2] Microvision, Inc. Redmond, WA [Online]. Available: http://www.microvision.com

[3] H. Urey, "MEMS scanners for display and imaging applications," Proc. SPIE-Int. Soc. Opt. Eng., vol. 5604, pp. 218-229, Jan. 2004.

[4] Y. C. Ko, J. W. Cho, Y. K. Mun, H. G. Jeong, W. K. Choi, J. H. Lee, J. W. Kim, J. B. Yoo, and J. H. Lee, "Eye-type scanning mirror with dual vertical combs for laser display," Proc. SPIE_Int. Soc. Opt. Eng., vol. 5721, pp. 14-22, Jan. 2005.

[5] M. Yoda, K. Isamoto, C. Chong, H. Ito, A. Murata, S. Kamisuki, M. Atobe, and H. Toshiyoshi, "A MEMS 1D optical scanner for laser projection display using self-assembled vertical combs and scan-angle magnifying mechanism," in Proc. 13th Int. Conf. Solid-State Sens., Actuators Microsyst. (TRANSDUCERS), Jun. 2005, vol. 1, pp. 968-971.

[6] Y. Haga and M. Esashi, "Biomedical microsystems for minimally invasive diagnosis and treatment," Proc. IEEE, vol. 92, no. 1, pp. 98-114, Jan. 2004.

[7] U. Hofmann, S. Muehlmann, M. Witt, K. Dörschel, R. Schütz, and B. Wagner, "Electrostatically driven micromirrors for a miniaturized confocal laser scanning microscope," Proc. SPIE_Int. Soc. Opt. Eng., vol. 3878, pp. 29-38, Sep. 1999.

[8] H. Miyajima, M. Nishio, Y. Kamiya, M. Ogata, and Y. Sakai, "Development of two dimensional scanner-on-scanner for confocal laser scanning microscope LEXT series," in Proc. IEEE/LEOS Int. Conf. Opt. MEMS Their Appl., Oulu, Finland, Aug. 2005, pp. 23-24.

[9] L. Zhou, M. Last, V. Milanovic, J. M. Khan, and K. S. J. Pister, “Twoaxis scanning mirror for free-space optical communication between UAVs," in Proc. IEEE/LEOS Int. Conf. Opt. MEMS Their Appl., Aug. 2003, pp. 157-158.

[10] R. A. Conant, J. T. Nee, K. Y. Lau, and R. S. Muller, "A flat high-frequency scanning micromirror," in Proc. Hilton Head Solid-State Sens. Actuator Workshop, Hilton Head, SC, Jun. 2000, pp. 6-9.

[11] P. R. Patterson, D. Hah, H. Nguyen, H. Toshiyoshi, R. Chao, and M. C. Wu, "A scanning micromirror with angular comb drive actuation," in Proc. Int. Conf. Micro Electro Mech. Syst., Las Vegas, NV, Jan. 2002 pp. 544-547. 
[12] J. Kim, H. Choo, L. Lin, and R. S. Muller, "Microfabricated torsional actuator using self-aligned plastic deformation," in Proc. 12th Int. Conf. Solid-State Sens., Actuators Microsyst. (TRANSDUCERS), Boston, MA, Jun. 2002, pp. 1015-1018.

[13] D. T. McCormick and N. C. Tien, "Multiple layer asymmetric vertical comb-drive actuated trussed scanning mirrors," in Proc. IEEE/LEOS Int. Conf. Opt. MEMS Their Appl., Aug. 2003, pp. 12-13.

[14] J. F. Bille, C. F. H. Harner, and F. H. Loesel, Aberration-Free Refractive Surgery, 2nd ed. New York: Springer-Verlag, pp. 182-182.

[15] J. Mansell and R. L. Byer, "Micromachined silicon deformable mirror," Proc. SPIE-Int. Soc. Opt. Eng., vol. 3353, Adaptive Optical System Technologies, pp. 896-901, 1998.

[16] R. Yeh, S. Hollar, and K. S. J. Pister, "Single mask, large force, and large displacement electrostatic linear inchworm motors," in Proc. Int. Conf. Micro Electro Mech. Syst., Interlaken, Switzerland, Jan. 2001, pp. 260-264.

[17] H. Choo, R. Kant, D. Garmire, J. Demmel, and R. S. Muller, "Fast, MEMS-based, phase-shifting interferometer," in Proc. Hilton Head Solid-State Sens. Actuator Workshop, Hilton Head, SC, Jun. 4-8, 2006, pp. 94-95.

[18] S. Timoshenko and J. N. Goodier, Theory of Elasticity, 2nd ed. New York: McGraw-Hill, pp. 278-278.

[19] A. C. Ugural, Mechanics of Materials. New York: McGraw-Hill, 1991.

[20] M. Mrochen, "Converting wavefronts into corrections," in Proc. 3rd Int. Congr. Wavefront Sens. Aberration-free Refractive Correction, Interlaken, Switzerland, Feb. 2002.

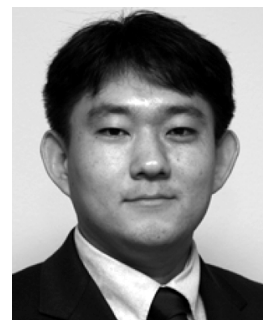

Hyuck Choo received the B.S. and M.Eng. degrees in 1996 and 1997, respectively, in electrical engineering from Cornell University, Ithaca, NY. He is currently working towards the Ph.D. degree in electrical engineering and computer sciences at the University of California, Berkeley (UC Berkeley), under Prof. R. S. Muller's supervision.

Before enrolling at UC Berkeley, he was with Kionix, Inc., Ithaca, NY, as a MEMS Test Engineer. $\mathrm{He}$ has focused his research on microlens and microscanner systems, particularly on applications to ocular-refractive surgery, biomedical-imaging systems, high-definition displays, and next-generation wavefront sensors. His most recent projects are directed toward the development of fast MEMS-based phase-shifting interferometers and their applications to the measurement of transient optical and biological phenomena.

Mr. Choo won the UC Berkeley EECS Lim Prize in 2001 (Best Performance on the Pre-Doctoral Exam) and is the two-time winner of the Berkeley Sensor and Actuator Center presentation award.

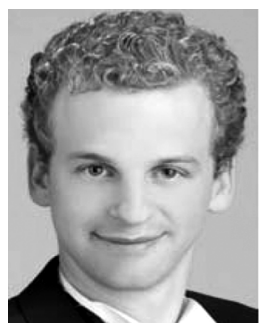

David Garmire received the B.S. degrees in both computer science and mathematics from Carnegie Mellon University, Pittsburg, PA. He is currently working towards the Ph.D. degree in computer science at the University of California, Berkeley (UC Berkeley) under the guidance of Prof. J. Demmel.

His main research area is in scientific computing focusing on MEMS simulation platforms, MEMS metrology techniques, and precision-control/sensing of MEMS devices using computers and high-speed analog/digital electronics. He has three United States international patents pending in MEMS photonics and sensors.

Mr. Garmire currently holds a Siebel Scholars Fellowship.

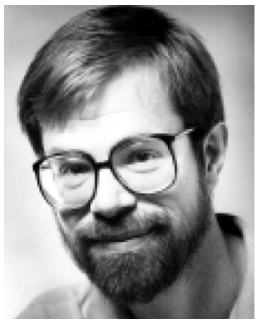

James Demmel (M'86-SM'00-F'02) is the Dr. Richard Carl Dehmel Distinguished Professor of Computer Science and Mathematics at the University of California, Berkeley (UC Berkeley). His personal research interests are in numerical linear algebra, high-performance computing, computer-aided design for MEMS, and applications of information technology to solve societal scale problems. He is best known for his work on the LAPACK and ScaLAPACK linear algebra libraries.

Prof. Demmel is a Fellow of the Association for Computing Machinery (ACM) and Member of the National Academy of Engineering. He is a winner of the Society for Industrial and Applied Mathematics (SIAM) J. H. Wilkinson Prize in Numerical Analysis and Scientific Computing, He was an invited speaker at the 2002 International Congress of Mathematicians and the 2003 International Congress on Industrial and Applied Mathematics.

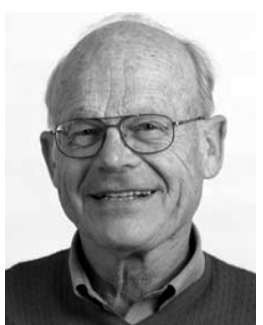

Richard S. Muller (S'57-M'58-SM'70-F'88LF'97) received the mechanical engineer's degree from Stevens Institute of Technology, Hoboken, NJ, the M.S. degree in electrical engineering and the Ph.D. degree in electrical engineering and physics, in 1962, from the California Institute of Technology, Pasadena.

After employment as a Member of the Technical Staff at Hughes Aircraft Company, he joined the faculty at the University of California, Berkeley, where he concentrated his research on the physics of integrated-circuit devices. Together with Dr. T. I. Kamins of Hewlett-Packard Company, he first published, Device Electronics for Integrated Circuits in 1977. A third edition of this book (which has been translated into five languages) was published in 2003. In the late 1970s, he began research in the area now known as MEMS and, together with R. M. White, he founded the Berkeley Sensor and Actuator Center in 1986. His present research focus is on optical MEMS. His present research focus is on optical MEMS.

Dr. Muller wrote the proposal to establish the IEEE/ASME JouRnAL OF MicROELECTROMECHANICAL SYSTEMS (JMEMS), of which he is now the Editor-in-Chief. A member of the U.S. National Academy of Engineering, he received a career MEMS Award at TRANSDUCERS' 97 as well as the IEEE Brunetti Award (1998, with R. T. Howe), a Fulbright Professorship, and a von Humboldt Research Award at Technische Universität Berlin (TU), Berlin, Germany, in 1994. His other awards include the Berkeley Citation and the Renaissance Award from Stevens Institute of Technology, where he served as a Trustee from 1996 to 2005. He has been a member of the National Materials Advisory Board and served on several National Research Council study panels as well as chairing a 1997 panel for which he acted as Editor of a widely distributed report on the promises and challenges of MEMS. He is a Member of the American Society of Mechanical Engineers (ASME). 